\title{
Article \\ Identification of an RNA-Binding-Protein-Based Prognostic Model for Ewing Sarcoma
}

\author{
Yi Chen ${ }^{1,2, *}\left(\mathbb{0}\right.$, Huafang $S u^{3}$, Yanhong Su ${ }^{1}$, Yifan Zhang ${ }^{1,2}$, Yingbo Lin ${ }^{1}$ and Felix Haglund ${ }^{1,2}$ \\ 1 Department of Oncology-Pathology, Karolinska Institutet, Solna, 17176 Stockholm, Sweden; \\ yanhong.su@ki.se (Y.S.); yifan.zhang@ki.se (Y.Z.); yingbo.lin@ki.se (Y.L.); felix.haglund@ki.se (F.H.) \\ 2 Clinical Pathology and Cancer Diagnostics, Karolinska University Hospital, Solna, 17176 Stockholm, Sweden \\ 3 Department of Radiation and Medical Oncology, The First Affiliated Hospital of Wenzhou Medical University, \\ Wenzhou 325000, China; suhuafang@wzhospital.cn \\ * Correspondence: yi.chen@ki.se
}

Citation: Chen, Y.; Su, H.; Su, Y.; Zhang, Y.; Lin, Y.; Haglund, F. Identification of an RNA-Binding-Protein-Based Prognostic Model for Ewing Sarcoma. Cancers 2021, 13, 3736. https:// doi.org/10.3390/cancers13153736

Academic Editors: Saurabh Agarwal and Jianhua Yang

Received: 28 May 2021

Accepted: 23 July 2021

Published: 25 July 2021

Publisher's Note: MDPI stays neutral with regard to jurisdictional claims in published maps and institutional affiliations.

Copyright: (C) 2021 by the authors. Licensee MDPI, Basel, Switzerland. This article is an open access article distributed under the terms and conditions of the Creative Commons Attribution (CC BY) license (https:// creativecommons.org/licenses/by/ $4.0 /)$.
Simple Summary: Ewing sarcoma (ES) is an aggressive childhood tumor for which response to chemotherapy is central to long-term prognosis, but few prognostic markers have been identified. RNA-binding proteins (RBPs) are strong regulators of cell behavior, working, for example, through post-translational modifications of mRNA. In this study, we investigated whether patterns in the RBP levels were related to outcomes in ES patients. A total of three distinct patterns were recognized, and additional modelling suggested that 10 RPBs had predictive value, suggesting that this model could be used in a clinical setting to identify patients with a higher risk of mortality.

Abstract: RNA-binding proteins (RBPs) are important transcriptomic regulators and may be important in tumorigenesis. Here, we sought to investigate the clinical impact of RBPs for patients with Ewing sarcoma (ES). ES transcriptome signatures were characterized from four previously published cohorts and grouped into new training and validation cohorts. A total of three distinct subtypes were identified and compared for differences in patient prognosis and RBP signatures. Next, univariate Cox and Lasso regression models were used to identify hub prognosis-related RBPs and construct a prognostic risk model, and prediction capacity was assessed through time-dependent receiver operating characteristics (ROCs), Kaplan-Meier curves, and nomograms. Across the three RBP subtypes, 29 significant prognostic-associated RBP genes were identified, of which 10 were used to build and validate an RBP-associated prognostic risk model (RPRM) that had a stable predictive value and could be considered valuable for clinical risk-stratification of ES. A comparison with immunohistochemistry validation showed a significant association between overall survival and NSUN7 immunoreactivity, which was an independent favorable prognostic marker. The association of RBP signatures with ES clinical prognosis provides a strong rationale for further investigation into RBPs molecular mechanisms.

Keywords: Ewing sarcoma; RNA-binding proteins; regulation network; prognosis prediction; risk model

\section{Introduction}

Ewing sarcoma (ES) represents the second-most common primary bone malignancy affecting children and adolescents, with an incidence of 2.9 per million/year [1-3]. It is an aggressive tumor typically characterized by a fusion of the Ewing sarcoma breakpoint region 1 (EWSR1) with an erythroblast transformation specific (ETS) transcription factor gene, most frequently (>95\%) the friend leukemia virus integration 1 (FLI1) gene [4,5]. Most types of ES harbors the $t(11 ; 22)$ (q24;q12) chromosomal translocation leading to the EWS-FLI1 fusion transcript. Genome-wide association studies identifying molecular features and genetic profiles suggest a generally low mutational burden for ES [6-8]. The STAG2 gene is the most frequently mutated (17\%) compared to normal paired cases, 
followed by CDKN2A (12\%), TP53 (7\%), and EZH2 (2.7\%). Genetic lesions of STAG2 and TP53 and deletions of CDKN2A are mutually exclusive and associated with the worst ES prognosis $[6,9,10]$. Recently, the Euro-Ewing 99 clinical trial revealed 3-year and 8-year overall survival (OS) rates of $72-78 \%$ and $56-65 \%$, respectively [11]. Patients with relapsed and advanced ES have a dismal prognosis, especially when the disease has metastasized, and a short survival time is expected [12]. Therefore, prognostic biomarkers and novel intervention targets are urgently needed.

RNA-binding proteins (RBPs) are a class of proteins that interact with a series of RNAs: messenger (mRNAs), ribosomal (rRNAs), non-coding (ncRNAs), micro (miRNAs), transfer (tRNAs), small nuclear (snRNAs), and small nucleolar (snoRNAs). They exert their functions by associating with their RNA targets and generating ribonucleoprotein complexes that regulate transcription, RNA maturation, translation, and metabolism and maintain post-transcriptional genome integrity [13-17]. Currently, more than $1500 \mathrm{RBPs}$ have been identified by large-scale quantitative methods accounting for about $7.5 \%$ of all protein-coding genes in the human genome $[18,19]$. EWS is a multifaceted RBP that has been implicated in modulating transcription, pre-mRNA splicing, and, importantly, in causing epigenetic remodeling in the tumorigenesis of ES [20,21]. Another example is IGF2BP3, a novel post-transcriptional regulator responsible for poor survival probability [22,23]. Our group previously reported that a higher expression of RBPs related to rRNA metabolism and mRNA splicing were significantly overrepresented in ES patients who had first-line treatment failure [22]. Thus, we hypothesized that a systematic study to explore prognosis-related RBPs would increase our knowledge of treatment resistance and facilitate risk-stratification.

In this study, we obtained ES gene expression data from previously published GEO datasets and investigated the RBP landscape in ES associated with patient outcomes. Next, the prognostic value of a 10-RBP expression signature was validated in an independent cohort. We also identified NSUN7 as a novel independent prognostic factor that could be a diagnostic and therapeutic target.

\section{Material and Methods}

\subsection{ES Data Processing}

Both the ES gene expression microarray data and clinical data were derived from the NCBI Gene Expression Omnibus (GEO) database: GSE63155 (46 ES cases, follow-up time: median 1876 days, range 155-3987 days) [24], GSE63166 (39 ES cases, follow-up time: median 2085 days, range 286-4500 days) [24], GSE17679 (32 ES cases, follow-up time: median 1159 days, range 138-5766 days) [25], and GSE34620 (38 ES cases, follow-up time: median 1549 days, range 270-4017 days) [26]. The patient characteristics involved in this study are shown in Table 1. In these four datasets, Affymetrix HuEx1.0 (GPL5175) and Affymetrix HG-U133 Plus 2.0 (GPL570) platform data were merged into one integrated dataset. Batch effects were removed using the ComBat function of the $R$ package surrogate variable analysis (sva) [27], and expression values were quantile-normalized across different samples. A total of 85patients from GSE63155 and GSE63166 were involved in the training cohort, while 70 from GSE17679 and GSE34620 were included in the validation cohort. We obtained a comprehensive RBP catalog consisting of 1542 genes and 318 transcriptional factors (TFs) for further analysis $[19,28]$. Unsupervised clustering was performed using the $\mathrm{R}$ package ConsesusClusterPlus to classify patients into distinct subtypes according to RBP expression. 
Table 1. GEO datasets and patient characteristics of ES.

\begin{tabular}{|c|c|c|c|c|c|c|c|c|}
\hline \multirow{2}{*}{ GEO ID } & \multirow{2}{*}{ No. of ES Cases Included } & \multirow{2}{*}{ Platform } & \multicolumn{2}{|c|}{ Age } & \multicolumn{2}{|c|}{ Sex } & \multicolumn{2}{|c|}{ Outcomes } \\
\hline & & & $<18$ & $\geq 18$ & Male & Female & Dead & Alive \\
\hline GSE63155 & 46 & HuEx1.0 (GPL5175) & 41 & 5 & 27 & 19 & 14 & 32 \\
\hline GSE63166 & 39 & HuEx1.0 (GPL5175) & 31 & 8 & 19 & 20 & 11 & 28 \\
\hline GSE17679 & 32 & U133Plus2.0 (GPL570) & 16 & 16 & 22 & 10 & 20 & 12 \\
\hline GSE34620 & 38 & U133Plus2.0 (GPL570) & 27 & 11 & 20 & 18 & 21 & 17 \\
\hline
\end{tabular}

\subsection{Identification of Differentially Expressed RBPs}

The R package linear models for microarray data (LIMMA) [29] was used to perform differentially expressed RBP (DERBP) analysis in the training cohort by comparing clusters of patient subtypes that were significantly associated with overall survival. A false discovery rate (FDR) of $<0.05$ or $p$-value of $<0.05$ was set as the cut-off criterion. A Venn diagram was used to determine the overlapping DERBPs between two sets of comparisons. Additionally, the DETFs and DEHallmarks were generated based on the same criteria.

\subsection{Protein-Protein Interaction (PPI) Network Construction and Functional Enrichment Analyses}

To further explore the potential molecular functions, the DERBPs were submitted into the Search Tool for the Retrieval of Interacting Genes (STRING) database for building the protein-protein interaction (PPI) network [30]. The PPIs with combined scores $\geq 0.7$ were selected, after which the network was constructed and visualized using Cytoscape software (version 3.8.2) (National Institute of General Medical Sciences, Bethesda, MD, USA) [31]. Any genes with a connectivity of $\geq 1$ (node/edge) were screened as hub genes for downstream analyses. The top 50 central nodes were ranked by The MCC (maximum clique centrality) algorithm of the CytoHubba plug-in [32] in Cytoscape. The functional enrichment analyses were performed using the ClueGO and CluePedia plug-ins in Cytoscape [33,34]. The main parameters of the constructing network with ClueGO were as follows: marker list, homo sapiens; ontologies/pathways, GO (biological process) and REACTOME pathways; GO tree interval, level = 6; GO term/pathway selection, $\mathrm{min}$ \# genes $=6$ and $\%$ genes = 6.000; and GO term/pathway network connectivity (kappa score $)=0.45$. Only GO terms $/$ pathways with $p<0.01$ were selected.

\subsection{Prognosis-Related RBPs and the Interaction with TFS}

After combining the expression levels of the hub genes with the survival status and follow-up time, a univariate Cox proportional regression analysis was used to determine the hub genes related to overall survival in the training cohort. The association between the expression level of significant survival-related genes and DETFs was assessed by Pearson correlation analysis. A correlation coefficient of $\geq 0.4$ and $p$-value of $\leq 0.05$ were set as the cut-off criteria. The Cytoscape ClusterViz plug-in [35] was employed to identify biological network modules between RBPs and TFs.

\subsection{Establishment and Validation of an RBP-Associated Prognostic Risk Model (RPRM)}

The survival-related genes were subjected to penalized multivariate Cox proportional hazard survival modeling by an algorithm for variable selection based on L1-penalized Least Absolute Shrinkage and Selection Operator (LASSO) regression [36]. The process of prognostic model construction was repeated for the combination of parameter values for 1000 iterations. Subsequently, the resulting models were combined through crossvalidation. The risk score formula for each sample was calculated as follows:

$$
\text { Risk score }=\beta 1 * \operatorname{Exp} 1+\beta 2 * \operatorname{Exp} 2+\beta i * \text { Expi }
$$

where $\beta$ represents the coefficient value, and Exp represents the gene expression level. Subsequently, A time-dependent receiver operating characteristic (ROC) curve was per- 
formed to evaluate the model performance by calculating the area under the curve (AUC). As a result, patients were classified into high- or low-risk groups according to the median risk score. The RBP-associated prognostic risk model (RPRM) was externally validated by the validation cohort (GSE17679 and GSE34620) to access its generalizability. Afterwards, we developed a nomogram-combing gene expression to improve risk stratification and quantify the risk assessment for survival probability at one year, three years, and five years in individual patients.

\subsection{Assessment of Gene Expression Level and Prognostic Significance in RPRM}

Our group had previously identified several pathways that were significantly associated with first-line treatment failure in ES [22]. Here, a single sample gene set enrichment analysis (ssGSEA) was used to define the estimated enrichment score of the gene signature for each sample using gene set variation analysis (GSVA) in R package [37]. Hallmark gene sets were downloaded from the Molecular Signatures Database v7.4. Gene expression levels in RPRM were assessed between dead and live patients. Significant genes were cross-validated by the validation cohort and our ES RNA-seq data.

\subsection{Immunohistochemistry}

We investigated the expression of NSUN7 in ES tumor tissue from patients $(n=24)$ who underwent standard treatment protocols (ISG/SSG III or EuroEwing99/2012 protocols) [38]. These were therapy-naive preoperative biopsies $(n=9)$, but if these were unavailable for analysis, viable tumor cells from a resection specimen $(n=11)$ or metastatic specimen $(n=4)$ were used.

Immunohistochemical staining for NSUN7 was done using a manual protocol with a polyclonal antibody (anti-NSUN7, Product \#PA5-54257 from ThermoFisher Scientific, Waltham, MA, USA) at 1:50 dilution. Formalin-fixed paraffin embedded (FFPE) tissue was sectioned in 4 um thick sections and deparaffinized. Antigen retrieval was performed for $10 \mathrm{~min}$ in low $\mathrm{pH}$ (citrate). Slides were incubated with the primary antibody at $4{ }^{\circ} \mathrm{C}$ overnight, followed by incubation with a biotinylated secondary antibody and signal generation using the VECTASTAIN ${ }^{\circledR}$ Elite ABC-HRP Kit (Vector Laboratories, Burlingame, CA, USA).

Two clinical pathologists evaluated the staining, and after agreeing on a scoring method, the slides were scored independently in a blinded fashion. The agreement was substantial (82.6\%, Cohen's K 0.620). There was disagreement in four cases, but consensus was reached after a second review. The slides fit into three scoring categories:

- Negative ("Most of the tumor cells were completely negative"): $>75 \%$ completely negative, $<25 \%$ with very weak cytoplasmic staining);

- Weak ("Cases exhibited a diffuse weak staining with small areas with negative or stronger immunoreactivity"): $>75 \%$ of tumor cells exhibited weak immunoreactivity); and

- Moderate ("Cases showed stronger cytoplasmic immunoreactivity in most of the tumor cells, but smaller areas with either weaker or negative immunoreactivity were sometimes observed"): $>75 \%$ of the tumor cells showed moderate cytoplasmic reactivity.

Since the number of patients was limited, the staining groups were compared in two different fashions: all groups (negative vs. weak vs. moderate) or simplified (negative vs. positive).

\subsection{Statistical Analysis}

Normally distributed variables were analyzed with an unpaired two-tailed Student's $t$-test, whereas a Mann-Whitney U test or a Wilcoxon rank-sum test was used for nonnormally distributed variables. The relationship between the parameters was evaluated using the Spearman rank correlation coefficient. A log-rank test Kaplan-Meier curve and a Cox proportional hazard regression were performed for survival analysis using the $\mathrm{R}$ package "survminer" and "survival". Additionally, L1-penalized LASSO regression and ROC 
curves were conducted using the R package "glmnet" and "pROC", respectively [39-41]. R version 4.0.3 (R Foundation for Statistical Computing, Vienna, Austria) was used to execute all statistical tests and plots.

\section{Results}

\subsection{Data Preprocessing of the ES Dataset}

Data from 155 ES patients with complete follow-up and expression data from GSE61355, GSE63156, GSE34620, and GSE17679 were bioinformatically pretreated to remove batch effects. The 15,016 overlapping genes across the four GEO datasets are depicted in the Venn diagrams in Figure S1A. Data before and after normalization were carefully inspected by principal component analysis (PCA), suggesting the batch effect was successfully removed using ComBat (Figure S1B,C). In addition, the gene expression profiles were visualized in box plots to show the impact on batch effect removal (Figure S1D,E).

\subsection{Identification of DERBPs in ES Patients and Transcriptional Subtypes}

To investigate how RBPs affect the prognostic significance in ES, we compared the gene expression between dead and alive patients using the Wilcoxon rank-sum test $(p<0.05)$ in the training cohort. This analysis identified 22 DERBPs, and the consensus k-mean clustering on the 22 RBP signatures clearly divided ES patients into three main subtypes with clustering stability decreasing for $\mathrm{k}=2$ to 6 (Figures $1 \mathrm{~A}$ and S2). They were clustered into RS1 $(n=32)$, RS2 $(n=42)$, and RS3 $(n=11)$ with distinct prognostic differences. Interestingly, patients in Cluster 1 had the best clinical prognosis, whereas patients in Cluster 3 had the poorest (log-ranktest, $p<0.05$ ) (Figure 1B). Following this, a heatmap visualized the expression levels of 22 RBPs in three RBP subtypes with survival status, where most were RS3s dominated by down-regulated transcripts (Figure 1C). Among 1542 RBPs quantified in 85 ES patients with two comparisons (RS1 vs. RS2, RS1 vs. RS3), $377(24.4 \%)$ were significantly down-regulated, and $374(24.3 \%)$ were significantly upregulated in RS3 compared to RS1; 150 (9.7\%) were significantly down-regulated, and 137 (8.9\%) were significantly up-regulated in RS2 compared to RS1 (Wilcoxon rank-sum test, FDR < 0.05; Figure 1D). A total of 90 overlapping up-regulated and 125 overlapping down-regulated DERBPs were identified by the two comparisons and selected for further analysis (Figure 1E).

\subsection{Functional Enrichment Analysis and PPI Network of DERBPS}

Among the DERBPs, we wanted to identify the key components using co-variation analysis. Functional enrichment analysis was performed for the 218 overlapping DERBPs: 90 up-regulated, 125 down-regulated, and 3 with distinct expression profiles. Gene ontology (GO) biological process and REACTOME pathway analyses were applied to clarify the DERBP gene function. As shown in Table S1, most of the DERBPs were enriched in the GO categories of RNA metabolism (i.e., RNA processing, RNA metabolic process, and RNA catabolic process) and protein metabolism (i.e., translation, ribonucleoprotein complex assembly). In addition, enrichment analysis of the REACTOME pathways revealed that RNA metabolism and mRNA splicing and translation were mostly enriched for DERBPs (Table S2). The GO term/pathway network is shown in Figure 2A,B $(p<0.01)$. Next, we built a high-confidence PPI network that contained 171 nodes (genes) and 1234 edges, which were selected for further analysis. To identify the top 50 hub nodes, the cytoHubba plug-in was applied, and key genes were selected from the PPI network according to the MCC score (Figure 2C). Detailed gene descriptions and connectivity degrees of the top 15 hub genes are shown in Table S3. EIF4A3 was identified as having the largest number of edges interacting with 54 other genes, followed by POLR2F and SRSF1. 
A

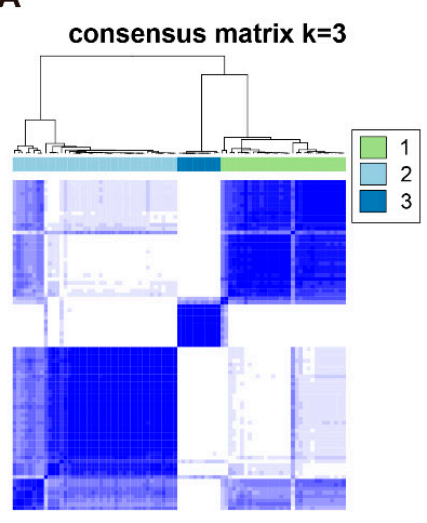

D

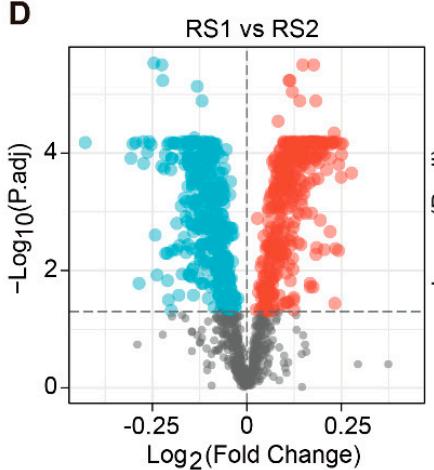

B

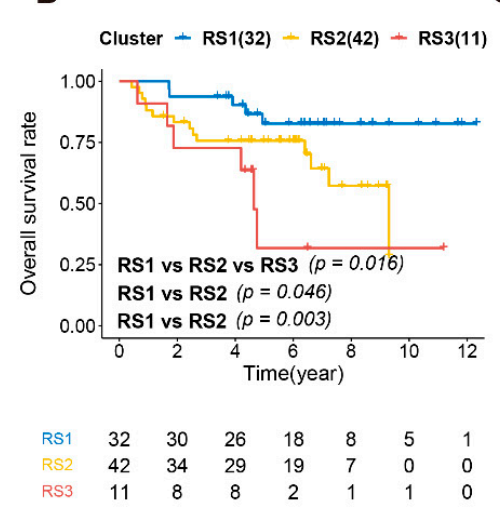

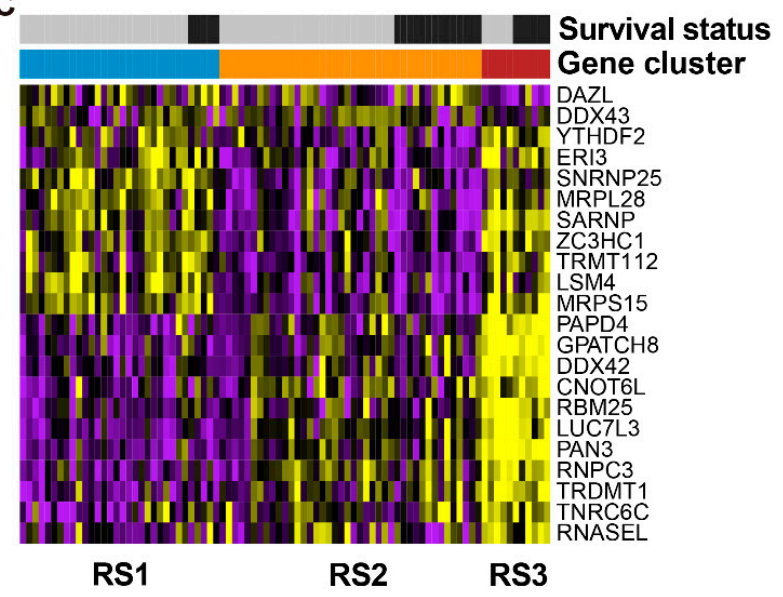
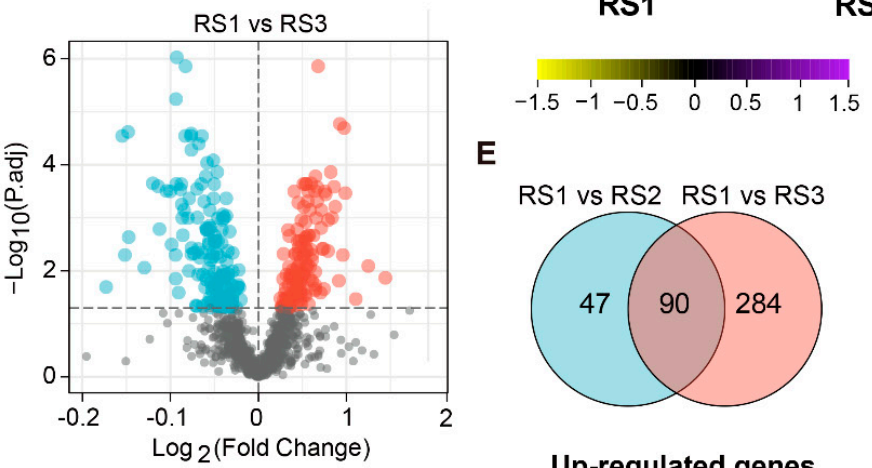

Survival status

E

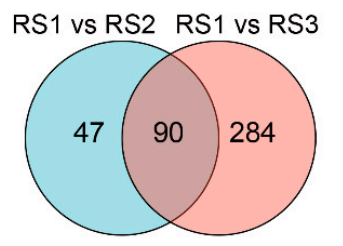

Up-regulated genes

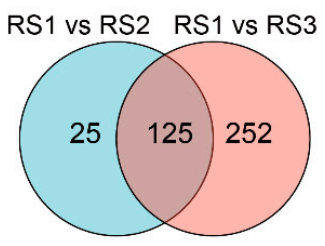

Down-regulated genes

Figure 1. DERBP identification and the transcriptional subtypes of ES (A). Consensus matrices of identified clusters $(\mathrm{k}=3)$. (B). Kaplan-Meier curves show the overall survival in ES patients among the three subtypes $(p=0.016, p=0.045, p=0.003$ for RS1 vs. RS2 vs. RS3, RS1 vs. RS2 and RS1 vs. RS3, respectively). (C). Abundances of 22 DERBPs (identified in dead ES patients) in the three RSs. (D). Expression profile of RBPs between RS1 versus RS2 and RS1 versus RS3, respectively. Red and blue dots represent upregulated and downregulated RBPs in RS2 or RS3, respectively (FDR < 0.05). (E). Overlap up-regulated and down-regulated DEREPs between RS1 versus RS2 and RS1 versus RS3.

\subsection{Prognosis-Related RPBs and the Regulatory Network}

To investigate the prognostic significance of these 171 RBPs involved in the PPI network, a univariate Cox regression analysis was performed from which 29 prognosticassociated hub RBP genes were obtained: 12 having favorable factors with a hazard ratio (HR) of $<1$, and 17 having risk factors (HR $>1$ ) (Figure $3 \mathrm{~A}$ ).

Next, we identified DETFs across the three RSs to explore the regulatory mechanisms of these RPBs. We found 144 TFs significantly expressed in RS3 compared to RS1, and 28 TFs significantly expressed in RS2 compared to RS1 (FDR < 0.05). The overlapping 18 DETFs in the two comparisons were then selected (Figure 3B). A heatmap was constructed to indicate their expression levels in three subtypes with survival status (Figure 3C). We consequently created a regulatory network based on these $18 \mathrm{TFs}$ and our 29 prognosis-related RPBs. A correlation score of more than 0.4 and $p<0.01$ were set as the cut-off values. The TFbased regulatory network topology was grouped into three clusters through the ClusterViz plug-in in Cytoscape. Notably, the schematic clearly illustrated the regulatory relationships among these TFs and RBPs (Figure 3D, Table S4). 
A

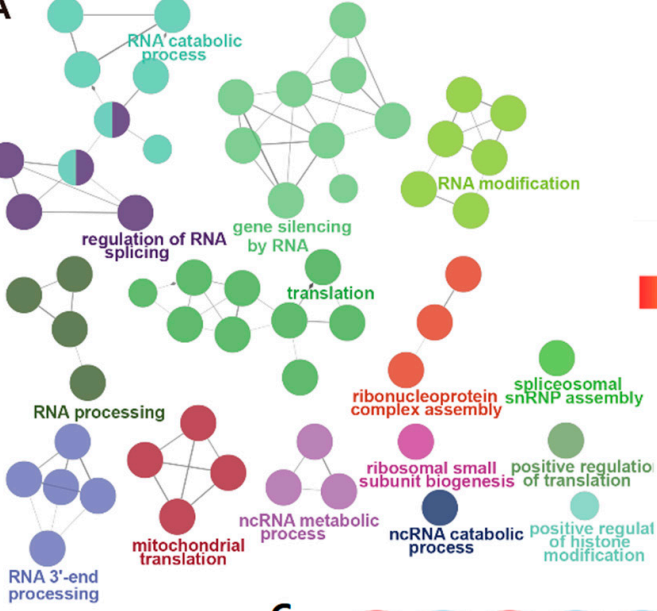

Node size significance

- $p V>0.1$

o $p V 0.05-0.1$

p $p$ 0.005-0.05

pV 0.0005-0.005

$p V<0.0005$

Group color

C CCAR1 CCNT2 CD2BP2 CLK1 CNOT4 CNOT6L CPSF4 CPSF6 CSTF3 CWF1912DCAF13 DCP1B DDX17 DDX23

DDX42 DDX49 DDX54 EED EIF3G EIF4A2 EIF4A3 EIF4E2 EIF4E3 ERAL1 FBL GEMIN8GPATCHIPATCH8 GUF1 HNRNPH3 INTS2 INTS4 INTS6 INTSB LSM2 LSM4) LSM7 LUC7L3 MEPCE METTL1METLL14MRPL11

Top 50 nodes

MRPL12 MRPL14 MRPL15 MRPL17 MRPL28 MRPL37 MRPL40 MRPL9 MRPS11 MRPS12 MRPS2 MRPS34 MRRF MTIF3

MTRF1 NAT10 NOP10 PAN3 PCBP2 PCF11 PIH1D1 PINX1 POLR2E POLR2F POLR2G POLR2I POLR2L PPIL4

PPWD1 PRPF18 PRPF19 PRPF39 PRPF4B RALY RAN RBBP6 RBM25 RBM39 RBM5 RNPC3 RNPS1 RPL15

RPL19 RPL6 RPMS17 RRP12 RUVBL2 SETX SF3B1 SF3B5 SNRNP25SNRPA1 SNRPB SNRPB2 SNRPF SREK1

SRP54 SRRT SRSF1 SRSF11 SRSF5 STRAP TCERG1 THOC1 THOC2 THOC6 TIA1 TIAL1 TOE1 TRMT112

Low MCC score

High MCC score

TSFM UPF2 UTP6 WBP4 XRN1 YTHDC1 YTHDC2 ZC3H6 ZCCHC6 ZFC3H1 ZMAT5 ZNF638 ZRANB2

Figure 2. Functional enrichment analysis of DERBPs and PPI Network (A). GO terms (biological process) grouped network (kappa score levels $\geq 0.45, p<0.01$ ). Ellipse represents the GO terms. The node size represents the significance of the term enrichment ( $p$-value), and the colors represent different functional groups. (B). The REACTOME pathway grouped network (kappa score levels $\geq 0.45, p<0.01$ ). Ellipse represents the GO terms. The node size represents the significance of the term enrichment ( $p$-value), and the colors represent different functional groups. (C). The MCC score of top 50 genes in the PPI network of DERBPs (combined score $\geq 0.7$ ) is represented by a red to yellow gradient.

\subsection{Construction and Validation of the RPBs-Associated Prognostic Risk Model (RPRM)}

After identifying the 29 prognosis-related RBPs, 1000 iterations of LASSO-penalized multivariate modeling were constructed, which led to 10 features with a non-zero, coefficientbased-risk model called RPRM (Figure 3E,F). We then computed the RPRM for each patient based on the risk score formula. The ROC curve exhibited significant prognostic performances for the AUC (1-year, 3-year, and 5-year OS predictions were 0.960, 0.915, and 0.817 in the training cohort and $0.745,0.700$, and 0.792 in the validation cohort, respectively (Figures $3 \mathrm{G}$ and $4 \mathrm{D}$ ). The samples in both the training and validation cohorts were subsequently separated into high- and low-risk groups according to the median risk score. Assessments of the survival of the two groups by Kaplan-Meier estimates showed that high-risk patients had a significantly worse overall survival than the low-risk patients in both cohorts $(p<0.001)$ (Figure $4 \mathrm{~A}, \mathrm{E})$. Moreover, the distribution of risk score, survival time, and survival status of each patient is illustrated in Figure 4B. Among the 10 significant genes in the risk model, NSUN7, RPL15, ZCCHC6, DCP1B, and GPATCH8 were associated with a favorable prognosis, while DDX23, STRAP, PRDX1, RPL6, and DCAF13 were considered to be risk factors (Figure $4 \mathrm{C}$ ). Subsequently, a nomogram combining these genes was generated, and each patient was assigned a series of scores corresponding to all of the 
involved genes. Then the 1-, 3-, and 5-year survival probabilities were projected to the final sum of the scores (Figure $4 \mathrm{E}$ ).

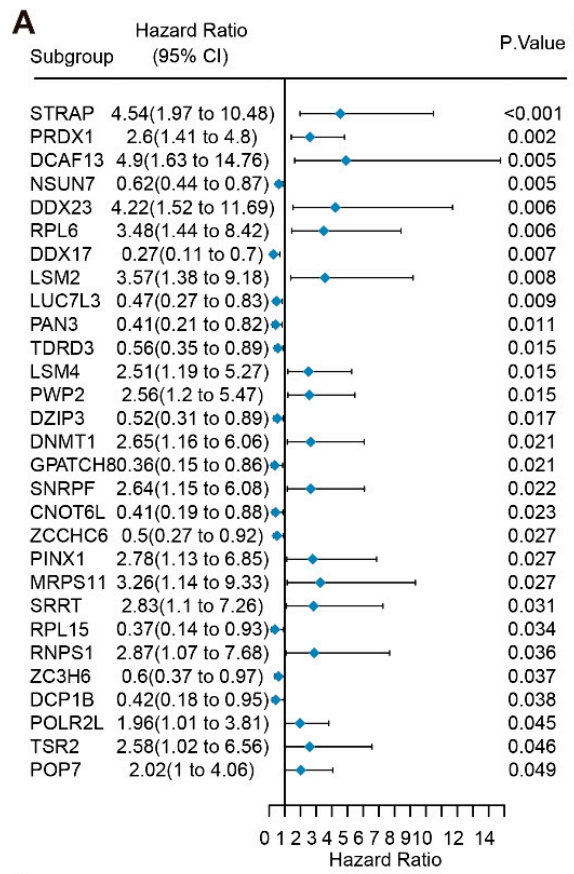

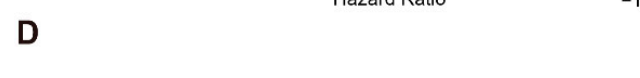

B
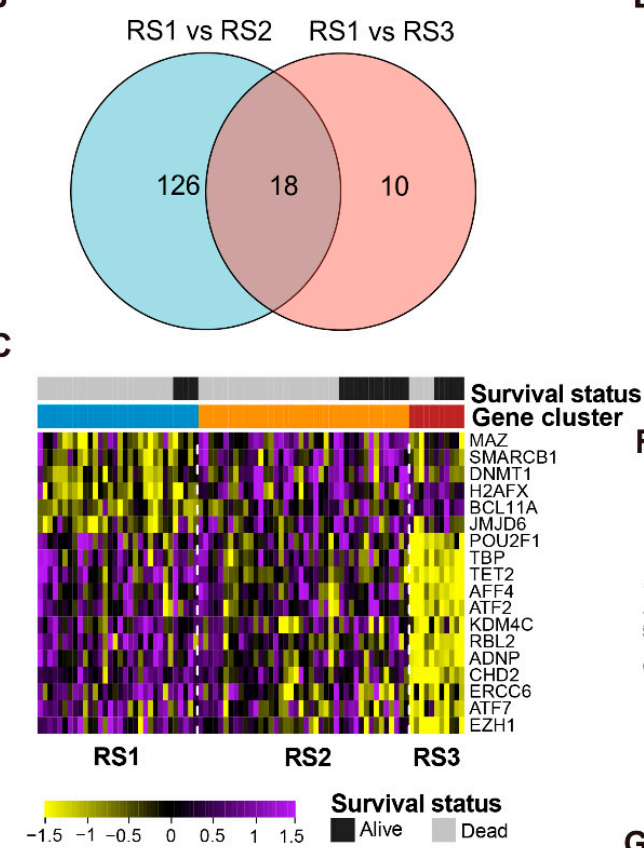

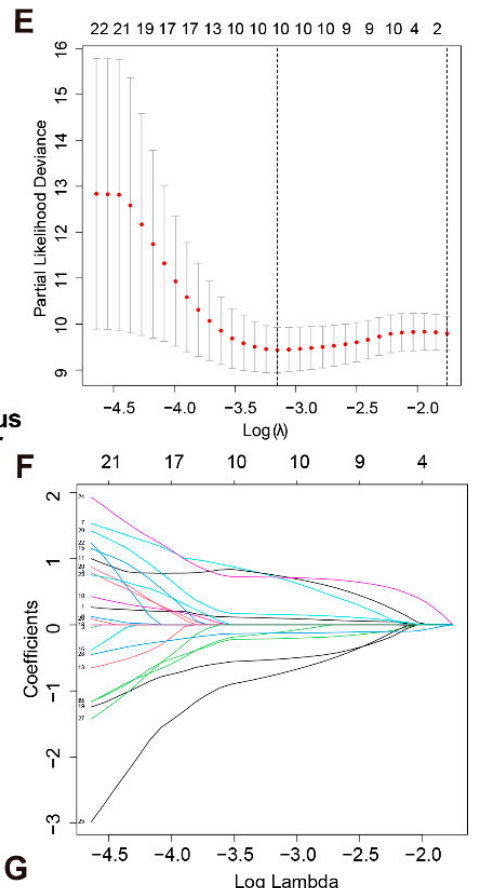

G

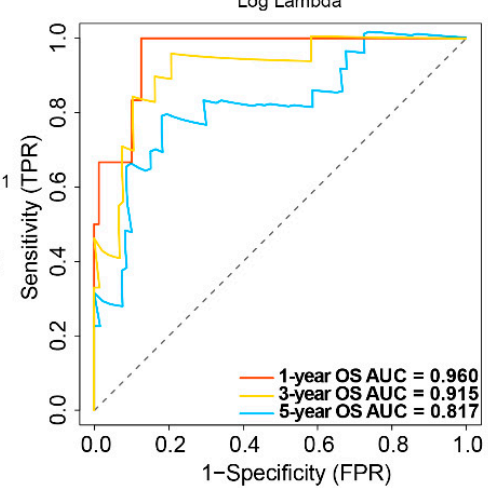

Figure 3. RBP regulatory network and prognostic risk model (A). Univariate analyses of 29 significant DERBPs with overall survival $(p<0.05)$. (B) Abundances of 18 DETFs (identified in dead ES patients) in the three RSs. (C) Overlap of DETFs between RS1 versus RS2 and RS1 versus RS3. (D). DERBPs network in ES. Circles represents all genes. Red indicates TF; blue indicates high risk RBP; and green indicates low risk RBP. The size of each circle indicates the degree of correlation. Grey lines indicate positive correlations, and purple lines indicate negative correlations $(\mathrm{r}>0.4, p<0.01)$. (E). Partial likelihood deviance under each log (lambda) was drawn in a LASSO Cox regression model. (F). The change trajectory of each independent variable in the model. $(G)$. ROC curve of the prognostic values of the RPRM risk model in training group in 1-, 3- and 5-year OS.

Since our group previously revealed that a series of pathways (e.g., apoptotic process, PI3K pathway, RNA splicing, rRNA metabolic process, glycolysis) were significantly associated with first-line treatment failure in ES patients, we performed an ssGSEA analysis in 50 hallmark gene sets to determine the relationship between pathway-enrichment and risk scores (Table S5). As shown in Figure 4G, 23 hallmark gene sets were significantly enriched between high- and low-risk patients, in which only protein secretion and bile-acid metabolism was positively associated with better prognosis. A total of ten overrepresenting gene sets are illustrated in Figure S3A $\left(-\log _{10}(\mathrm{FDR})>3\right)$. Notably, positive correlations between the risk and ssGSEA scores in high-risk patients were shown in 16 gene sets $(p<0.05)$ (Figure $4 \mathrm{H}$, Table S6), where the reactive oxygen species pathway, increased UV 
response, and glycolysis represent the top three highest correlation coefficients $(p<0.001$; $r=0.67,0.61$, and 0.60, respectively) (Figure S3B-D).
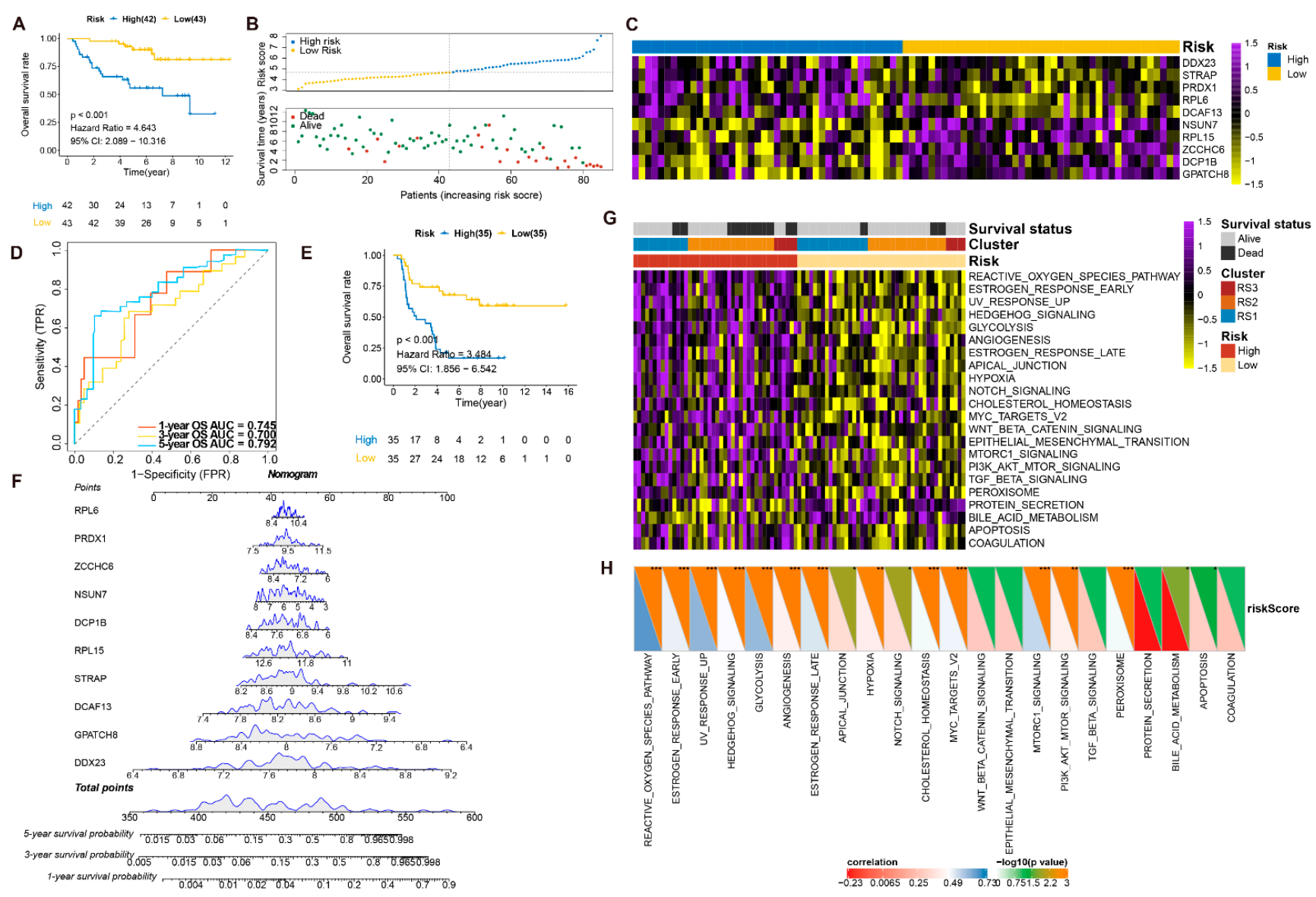

H

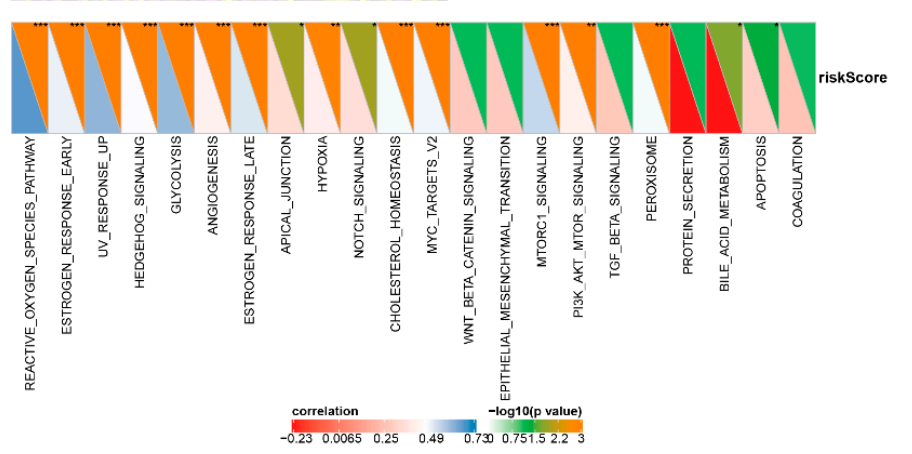

Figure 4. Validation and assessment of RPRM (A). Kaplan-Meier curves show overall survival between high-risk and low-risk patients in the training group $(p<0.001)$. (B). Distribution of risk score and survival time of patients in the training group. The patients were divided into high-risk and low-risk subgroups based on the median value of the risk score. Blue and yellow dots represent high- and low-risk patients, respectively. In the plot below, red and green dots indicate dead and live patients, respectively. (C). Abundances of 10 significant RBPs (involved in RPRM) in the training group. (D). ROC curve of the prognostic values of RPRM risk model in 1-, 3- and 5-year validation groups. (E). Kaplan-Meier curves show overall survival in validation groups between high- and low-risk patients $(p<0.001)$. (F). Nomogram for predicting $1-, 3-$, and 5-year survival probability of ES patients in the training group. The total score of these genes for each patient is on the total points axis, which corresponds to the survival probabilities plotted on the three axes below. (G). Abundances of 23 DEHallmarks between high- and low-risk patients in the three RSs. (H). The correlation between LDA score and enrichment scores of 23 DEHallmarks. ${ }^{*} p<0.05 ;{ }^{* *} p<0.01$, ${ }^{* * *} p<0.001$.

\subsection{Validation of the Prognostic Value and Expression of the RBPs Involved in RPRM}

To further assess the independent prognostic value of each key RBPs in ES, the KaplanMeier estimates were used to evaluate the relationship between these key RBPs and OS. As a result, six gene expressions (DCP18, DDX23, GRATCH8, NSUN7, RPL6, and ZCCHC6) were identified as being significantly associated with OS in the training cohort: high ( $\geq$ median) and low ( $<$ median). A total of four of these were beneficial to prognosis, and two were associated with poor outcomes $(p<0.05)$ (Figures 5A and S5). The Kaplan-Meier plots of the other remaining four genes are shown in Figure S4 $(p>0.05)$. Additionally, we found that the expression level of DDX23, RPL6, and NSUN7 were significantly correlated with survival status (Wilcoxon rank-sum test, $p<0.05$, Figure 5B), but only NSUN7 could be verified (log-rank test, Wilcoxon rank-sum test, $p<0.05$, Figure $5 C, D$ ). 
A

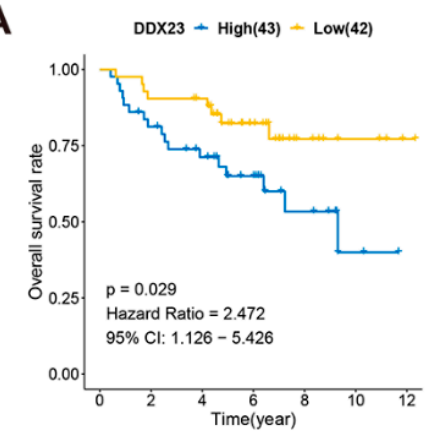

B

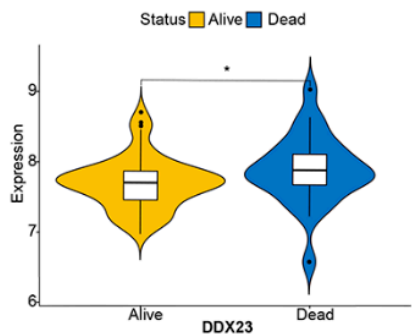

C

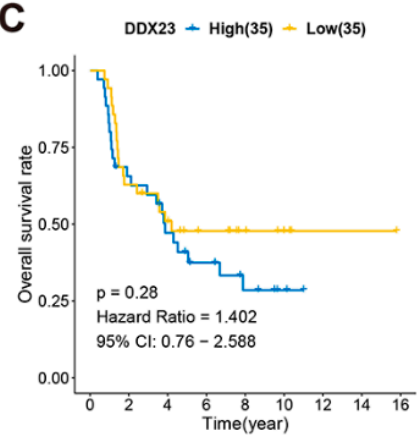

D

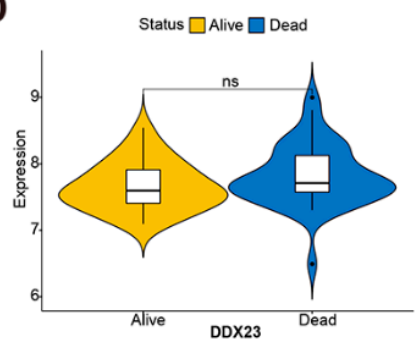

Training cohort

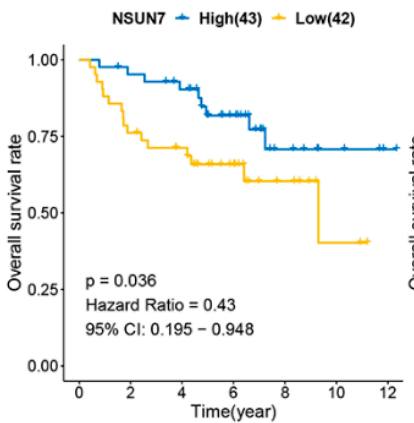

Status $\square$ Alive $\square$ Dead
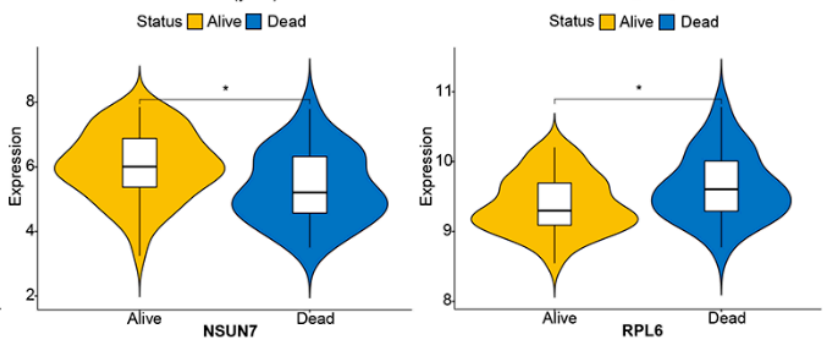

Validation cohort
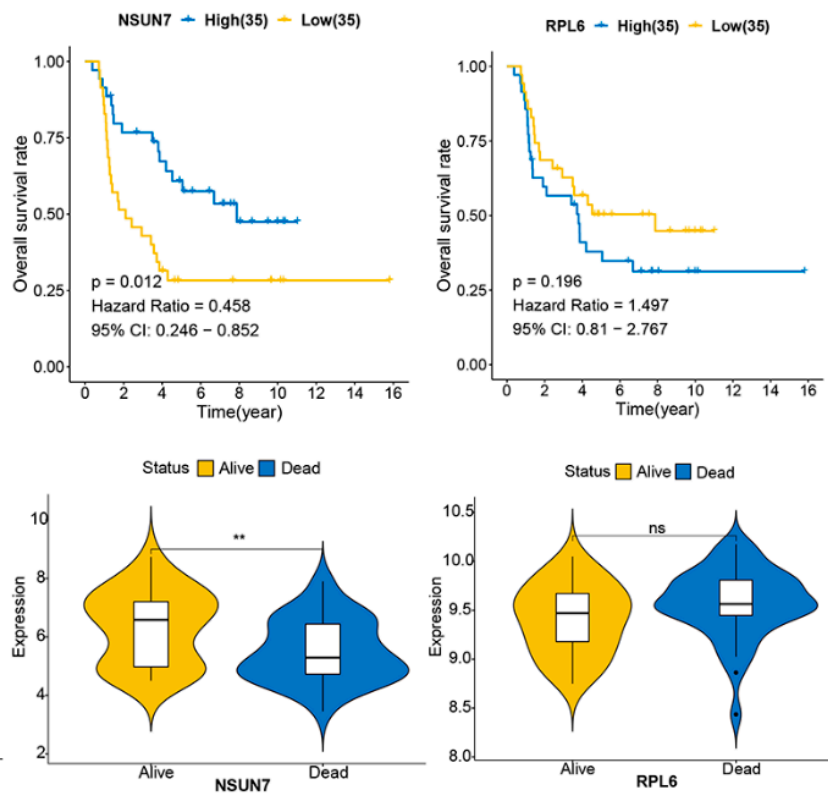

Figure 5. Validation of the prognostic and expression value of the RBPs involved in RPRM in training cohort (A). Kaplan-Meier curves show the overall survival in training group with highrisk and low-risk subgroups by DDX23, NSUN7, and RPL6, based on the median value of these genes, respectively. $(p<0.05)$. (B). The expression levels of three significant genes in the training group by survival status. The Wilcoxon rank sum test was used to compare the differences between groups. ${ }^{*} p<0.05$. (C). Kaplan-Meier curves show overall survival in validation group with high-risk and low-risk subgroups by DDX23, NSUN7, and RPL6 based on the median value of these genes, respectively. (D). The expression levels of DDX23, RPL6, NSUN7 in validation group by survival status. The Wilcoxon rank sum test was used to compare the differences between groups. ${ }^{* *} p<0.01$, ns: no significant.

The immunoreactivity of NSUN7 was scored as negative $(n=8)$, weak $(n=6)$ or moderate $(n=10)$ in 24 patients (Table S7). The proportion of negative cases was higher in pre-treated tissue $(7 / 15)$ than in therapy-naïve $(1 / 9)$ biopsies, which may indicate 
that NSUN7-negative cells are more resistant to treatment. In the therapy-naive biopsies, $5 / 9$ cases were classified as good responders according to the treatment protocol $(<10 \%$ viable tumor cells after treatment), and in the post-therapy specimens, only $2 / 11$ were good responders. We found a significant association between NSUN7 immunoreactivity and overall survival (Fisher's exact test, $p=0.0069$, Figure 6A) and that NSUN7 negative cases had a shorter overall survival compared to positive ones (log-rank test, $p=0.044$, Figure 6B). From the preoperative biopsies, only a single patient died (scored as negative for NSUN7). The prognostic association across three comparisons (negative vs. weak vs. moderate) are shown in Figure S6 and Figure 6C (chi-square test, $p=0.0084$; log-rank test, $p=0.101)$.

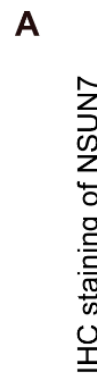

C

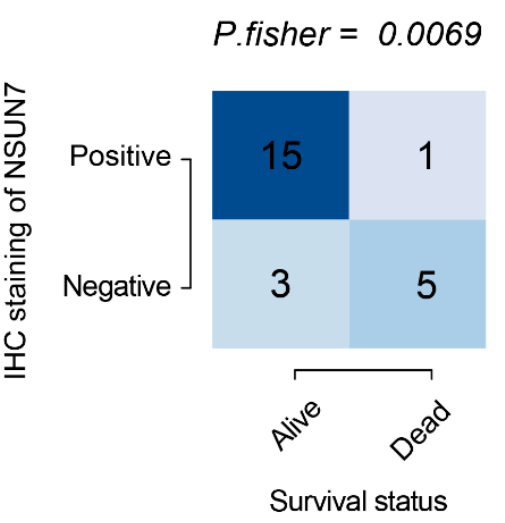

$100 \mathrm{X}$

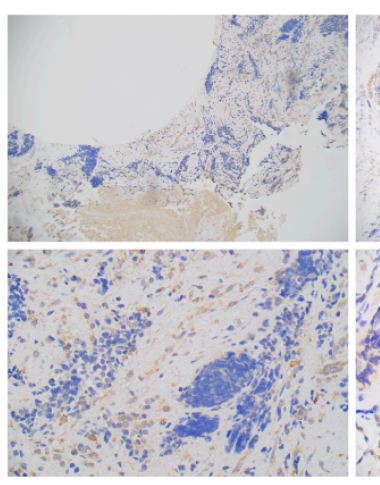

Negative
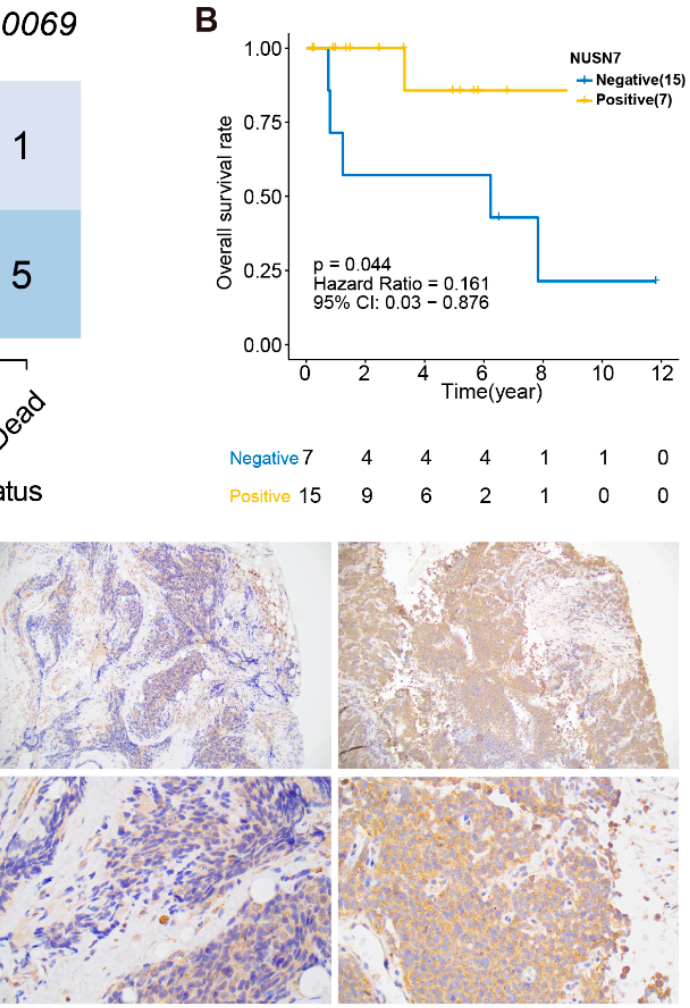

Weak

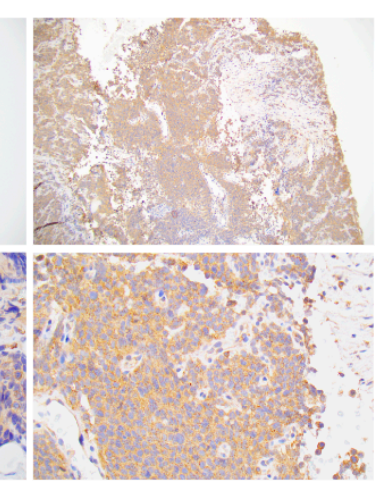

Moderate

Figure 6. Examples of histology imaging and follow-up in patients with ES. (A). Comparison of the survival status and the NSUN7 IHC scores in 24 ES patients (Fisher's exact test). (B). Kaplan-Meier curve shows the overall survival of NSUN7 positive and negative in the subgroup of IHC staining. (C). Comparison between NSUN7 immunoreactivity in negative, weak, and moderate scoring at $100 \times$ and $400 \times$ magnification.

These results strongly suggest that NSUN7 may be an important biomarker for ES therapy resistance and that the absence of immunoreactivity may be a prognostic marker.

\section{Discussion}

Our group had previously reported an association between RBP expression and treatment resistance in Ewing sarcoma [22]. Here, we sought to evaluate the putative relationship between RBPs and patient outcome in four previously published transcriptome datasets. We identified three RBP-related transcriptional subtypes that were significantly associated with overall patient survival. We constructed and validated a 10-gene prognostic risk model that showed good clinical applicability as a prognostic marker for ES patients. Finally, one individual transcript in the model (NSUN7) was validated on a protein level in a separate cohort. 
It is well known that RBPs are critical for various biological processes. Perhaps not surprisingly, we identified 171 prognostic-associated RBPs that were mostly enriched in RNA (mRNA, ncRNA, rRNA) metabolism, splicing, and translation, and these were similar to our previous findings from the RNA-sequencing of a single Ewing sarcoma cohort. Differential expression between the three RBP-related subtypes also identified 18 transcription factors that significantly co-varied with the 171 RBPs, potentially identifying the regulatory network that define the three RBP subtypes. While many of the identified transcription factors were described as central to cancer development (including SMARCB1, MAZ, EZH1 and H2AFX), none had an established role in ES, likely due to the limited number of functional studies of this tumor type.

Current chemotherapy protocols can cure a significant proportion of ES patients, but progression under chemotherapy is likely to drive resistance mechanisms. It may therefore be valuable to identify patients with a high likelihood of limited treatment response to offer alternative therapies in clinical trials. Since our 10-gene prognostic model was based on patients with current treatment regimes, our model could be used to identify patients who are at a high risk of death and who derive little benefit from current chemotherapy protocols.

When considering each gene in the model, only six were significantly associated with patient outcomes in the training cohort. The expression levels of three (DDX23, RPL6, and NSUN7) were related to survival status, but only NSUN7 was validated as an individual marker, which may be related to the semi-quantitative nature of immunohistochemistry protocols. While this may sound disheartening, it should be interpreted as meaning that multiple gene analyses are required to create a functional prognostic panel for ES. For comparison, validated and implemented prognostic panels for breast cancer contain $\sim 50$ genes [42].

Consistent with our previous findings, we also found that cancer-related pathways were mostly activated in the ES samples with high-risk scores. Of these, high expression of hypoxia-inducible factor $1 \alpha$ (HIF1 $\alpha$ ) emerged as an novel hallmark pathway, which was described to be correlated with a hypoxic tumor microenvironment in ES [43]. These findings are in line with the well-known prognostic value of serum lactate dehydrogenase (LDH) in ES patients [44].

\section{Conclusions}

In summary, this study identified three distinct RBPs signatures in ES. Through DERBP stratification, an RPRM risk model was constructed and validated to be a potential prognostic factor for ES patients. Major limitations of this study should be noted, including a relatively small sample size (155 patients) and the lack of detailed clinical characteristics for many of the cases. However, our results provide a strong rationale for the continued investigation of RBPs since they seem central to chemotherapy resistance and ES patient outcomes.

Supplementary Materials: The following are available online at https://www.mdpi.com/article/10 .3390 / cancers13153736/s1, Figure S1. GEO data processing and normalization (A). A Venn diagram illustrates the overlap of the genes across the four involved GEO datasets of ES. (B,C). The PCA plots show the overall GEO data profiles of GSE17679, GSE34620, GSE61355, and GSE61366 before and after removing batch effect, respectively. $(\mathrm{D}, \mathrm{E})$. The box plots show the expression profiles of GSE17679, GSE34620, GSE61355, and GSE61366 before and after removing batch effect, respectively. Figure S2. Consensus matrices of identified clusters $(k=2,4-6)$; Figure S3. (A). The distribution of significant gene sets enriched in high-risk patients at cutoff with $-\log 10$ (FDR) $>3$. (B-D). Violin plots show the correlation between risk score and enrichment scores of reactive oxygen species pathway, UV response up, and glycolysis, respectively. Figure S4. Kaplan-Meier curves show the overall survival in training group with high-risk and low-risk subgroups by DCF13, PRDX1, RPL15, and STRAP, based on the median value of these gene expression levels, respectively. $(p<0.05)$. Figure S5. (A). Kaplan-Meier curves show the overall survival in training group with high-risk and low-risk subgroups by DCP1B, GPATCH8, and ZCCHC6, based on the median value of these 
gene expression levels, respectively. $(p<0.05)$. (B). The expression levels of DCP1B, GPATCH8, and ZCCHC6 in training group by survival status. The Wilcoxon rank sum test was used to compare the differences between groups. ${ }^{*} p<0.05$. Figure S6. (A). Comparison of the survival status and the NSUN7 IHC scores (negative vs. weak vs. moderate) in 24 Ewing sarcoma patients (Fisher's exact test). (B). Kaplan-Meier curve show the overall survival of NSUN7 (negative vs. weak vs. moderate) in the subgroup of IHC staining. Table S1. The top 15 GO biological processes of DERBPs in ES. Table S2. The top 15 REACTOME pathways of DERBPs in ES. Table S3. The top 15 hub genes with the highest degree of connectivity in the PPI network. Table S4. Correlations between RBPs and TFs. Table S5. ssGSEA scores of 50 Hallmarks and risk score in training cohort. Table S6. The correlations between risk score and 23 significant hallmarks. Table S7. IHC scoring and corresponding clinical characteristics in 24 Ewing sarcoma patients' cohort.

Author Contributions: Conceptualization, Y.C., H.S., and F.H.; methodology, Y.C., H.S., and F.H.; software, Y.C.; validation, Y.C., Y.Z., Y.L., and F.H.; formal analysis, Y.C., H.S., Y.Z., Y.S., Y.L., and F.H.; investigation, Y.C., H.S., Y.S., Y.Z., Y.L., and F.H.; resources, Y.C. and F.H.; data curation, all authors; writing—original draft preparation, Y.C.; writing-review and editing, Y.C., H.S., Y.S., Y.Z., Y.L., and F.H.; visualization, Y.C., H.S., and F.H.; supervision, F.H.; project administration, Y.C. and F.H.; funding acquisition, F.H. All authors have read and agreed to the published version of the manuscript.

Funding: This work is supported by the Swedish Cancer Society, the Swedish Childhood Cancer Foundation, the Cancer Society in Stockholm, the Stockholm County Council, and the Karolinska Institutet.

Institutional Review Board Statement: The study was conducted according to the guidelines of the Declaration of Helsinki and approved by the Institutional Review Board (2017-1114).

Informed Consent Statement: Informed consent was obtained from all subjects involved in the study.

Data Availability Statement: The data presented in this study are available on request from the corresponding author.

Conflicts of Interest: The authors declare no conflict of interest.

\section{References}

1. Arndt, C.A.; Rose, P.S.; Folpe, A.L.; Laack, N.N. Common musculoskeletal tumors of childhood and adolescence. Mayo Clin. Proc. 2012, 87, 475-487. [CrossRef] [PubMed]

2. Esiashvili, N.; Goodman, M.; Marcus, R.B., Jr. Changes in incidence and survival of Ewing sarcoma patients over the past 3 decades: Surveillance Epidemiology and End Results data. J. Pediatr. Hematol. Oncol. 2008, 30, 425-430. [CrossRef] [PubMed]

3. Randall, R.L.; Lessnick, S.L.; Jones, K.B.; Gouw, L.G.; Cummings, J.E.; Cannon-Albright, L.; Schiffman, J.D. Is There a Predisposition Gene for Ewing's Sarcoma? J. Oncol. 2010, 2010, 397632. [CrossRef]

4. Delattre, O.; Zucman, J.; Plougastel, B.; Desmaze, C.; Melot, T.; Peter, M.; Kovar, H.; Joubert, I.; de Jong, P.; Rouleau, G.; et al. Gene fusion with an ETS DNA-binding domain caused by chromosome translocation in human tumours. Nature 1992, 359, 162-165. [CrossRef] [PubMed]

5. Sorensen, P.H.; Lessnick, S.L.; Lopez-Terrada, D.; Liu, X.F.; Triche, T.J.; Denny, C.T. A second Ewing's sarcoma translocation, $\mathrm{t}$ $(21 ; 22)$, fuses the EWS gene to another ETS-family transcription factor, ERG. Nat. Genet. 1994, 6, 146-151. [CrossRef] [PubMed]

6. Brohl, A.S.; Solomon, D.A.; Chang, W.; Wang, J.; Song, Y.; Sindiri, S.; Patidar, R.; Hurd, L.; Chen, L.; Shern, J.F.; et al. The genomic landscape of the Ewing Sarcoma family of tumors reveals recurrent STAG2 mutation. PLoS Genet. 2014, 10, e1004475. [CrossRef]

7. Crompton, B.D.; Stewart, C.; Taylor-Weiner, A.; Alexe, G.; Kurek, K.C.; Calicchio, M.L.; Kiezun, A.; Carter, S.L.; Shukla, S.A.; Mehta, S.S.; et al. The genomic landscape of pediatric Ewing sarcoma. Cancer Discov. 2014, 4, 1326-1341. [CrossRef]

8. Shukla, N.; Schiffman, J.; Reed, D.; Davis, I.J.; Womer, R.B.; Lessnick, S.L.; Lawlor, E.R.; The COG Ewing Sarcoma Biology Committee. Biomarkers in Ewing Sarcoma: The Promise and Challenge of Personalized Medicine. A Report from the Children's Oncology Group. Front. Oncol. 2013, 3, 141. [CrossRef]

9. Huang, H.Y.; Illei, P.B.; Zhao, Z.; Mazumdar, M.; Huvos, A.G.; Healey, J.H.; Wexler, L.H.; Gorlick, R.; Meyers, P.; Ladanyi, M. Ewing sarcomas with p53 mutation or p16/p14ARF homozygous deletion: A highly lethal subset associated with poor chemoresponse. J. Clin. Oncol. 2005, 23, 548-558. [CrossRef]

10. Kovar, H.; Auinger, A.; Jug, G.; Aryee, D.; Zoubek, A.; Salzer-Kuntschik, M.; Gadner, H. Narrow spectrum of infrequent p53 mutations and absence of MDM2 amplification in Ewing tumours. Oncogene 1993, 8, 2683-2690.

11. Whelan, J.; Le Deley, M.C.; Dirksen, U.; Le Teuff, G.; Brennan, B.; Gaspar, N.; Hawkins, D.S.; Amler, S.; Bauer, S.; Bielack, S.; et al. High-Dose Chemotherapy and Blood Autologous Stem-Cell Rescue Compared With Standard Chemotherapy in Localized High-Risk Ewing Sarcoma: Results of Euro-E.W.I.N.G.99 and Ewing-2008. J. Clin. Oncol. 2018, 36, 3110. [CrossRef] 
12. Lawlor, E.R.; Sorensen, P.H. Twenty Years on: What Do We Really Know about Ewing Sarcoma and What Is the Path Forward? Crit. Rev. Oncog. 2015, 20, 155-171. [CrossRef]

13. Fu, X.D.; Ares, M., Jr. Context-dependent control of alternative splicing by RNA-binding proteins. Nat. Rev. Genet. 2014, 15, 689-701. [CrossRef] [PubMed]

14. Nishida, K.; Kuwano, Y.; Nishikawa, T.; Masuda, K.; Rokutan, K. RNA Binding Proteins and Genome Integrity. Int. J. Mol. Sci. 2017, 18, 1341. [CrossRef]

15. Martin, K.C.; Ephrussi, A. mRNA localization: Gene expression in the spatial dimension. Cell 2009, 136, 719-730. [CrossRef] [PubMed]

16. Moore, M.J.; Proudfoot, N.J. Pre-mRNA processing reaches back to transcription and ahead to translation. Cell 2009, 136, 688-700. [CrossRef]

17. Sonenberg, N.; Hinnebusch, A.G. Regulation of translation initiation in eukaryotes: Mechanisms and biological targets. Cell 2009, 136, 731-745. [CrossRef]

18. Baltz, A.G.; Munschauer, M.; Schwanhausser, B.; Vasile, A.; Murakawa, Y.; Schueler, M.; Youngs, N.; Penfold-Brown, D.; Drew, K.; Milek, M.; et al. The mRNA-bound proteome and its global occupancy profile on protein-coding transcripts. Mol. Cell 2012, 46, 674-690. [CrossRef]

19. Gerstberger, S.; Hafner, M.; Tuschl, T. A census of human RNA-binding proteins. Nat. Rev. Genet. 2014, 15, 829-845. [CrossRef]

20. Araya, N.; Hirota, K.; Shimamoto, Y.; Miyagishi, M.; Yoshida, E.; Ishida, J.; Kaneko, S.; Kaneko, M.; Nakajima, T.; Fukamizu, A. Cooperative interaction of EWS with CREB-binding protein selectively activates hepatocyte nuclear factor 4-mediated transcription. J. Biol. Chem. 2003, 278, 5427-5432. [CrossRef] [PubMed]

21. Rossow, K.L.; Janknecht, R. The Ewing's sarcoma gene product functions as a transcriptional activator. Cancer Res. 2001, 61, 2690-2695.

22. Chen, Y.; Hesla, A.C.; Lin, Y.; Ghaderi, M.; Liu, M.; Yang, C.; Zhang, Y.; Tsagkozis, P.; Larsson, O.; Haglund, F. Transcriptome profiling of Ewing sarcomas-Treatment resistance pathways and IGF-dependency. Mol. Oncol. 2020, 14, 1101-1117. [CrossRef]

23. Mancarella, C.; Pasello, M.; Ventura, S.; Grilli, A.; Calzolari, L.; Toracchio, L.; Lollini, P.L.; Donati, D.M.; Picci, P.; Ferrari, S.; et al. Insulin-Like Growth Factor 2 mRNA-Binding Protein 3 is a Novel Post-Transcriptional Regulator of Ewing Sarcoma Malignancy. Clin. Cancer Res. 2018, 24, 3704-3716. [CrossRef]

24. Volchenboum, S.L.; Andrade, J.; Huang, L.; Barkauskas, D.A.; Krailo, M.; Womer, R.B.; Ranft, A.; Potratz, J.; Dirksen, U.; Triche, T.J.; et al. Gene Expression Profiling of Ewing Sarcoma Tumors Reveals the Prognostic Importance of Tumor-Stromal Interactions: A Report from the Children's Oncology Group. J. Pathol. Clin. Res. 2015, 1, 83-94. [CrossRef]

25. Savola, S.; Klami, A.; Myllykangas, S.; Manara, C.; Scotlandi, K.; Picci, P.; Knuutila, S.; Vakkila, J. High Expression of Complement Component 5 (C5) at Tumor Site Associates with Superior Survival in Ewing's Sarcoma Family of Tumour Patients. ISRN Oncol. 2011, 2011, 168712. [CrossRef]

26. Postel-Vinay, S.; Veron, A.S.; Tirode, F.; Pierron, G.; Reynaud, S.; Kovar, H.; Oberlin, O.; Lapouble, E.; Ballet, S.; Lucchesi, C.; et al. Common variants near TARDBP and EGR2 are associated with susceptibility to Ewing sarcoma. Nat. Genet. 2012, 44, 323-327. [CrossRef]

27. Leek, J.T.; Johnson, W.E.; Parker, H.S.; Jaffe, A.E.; Storey, J.D. The sva package for removing batch effects and other unwanted variation in high-throughput experiments. Bioinformatics 2012, 28, 882-883. [CrossRef] [PubMed]

28. Mei, S.; Meyer, C.A.; Zheng, R.; Qin, Q.; Wu, Q.; Jiang, P.; Li, B.; Shi, X.; Wang, B.; Fan, J.; et al. Cistrome Cancer: A Web Resource for Integrative Gene Regulation Modeling in Cancer. Cancer Res. 2017, 77, e19-e22. [CrossRef] [PubMed]

29. Ritchie, M.E.; Phipson, B.; Wu, D.; Hu, Y.; Law, C.W.; Shi, W.; Smyth, G.K. Limma powers differential expression analyses for RNA-sequencing and microarray studies. Nucleic Acids Res. 2015, 43, e47. [CrossRef]

30. Szklarczyk, D.; Gable, A.L.; Lyon, D.; Junge, A.; Wyder, S.; Huerta-Cepas, J.; Simonovic, M.; Doncheva, N.T.; Morris, J.H.; Bork, P.; et al. STRING v11: Protein-protein association networks with increased coverage, supporting functional discovery in genome-wide experimental datasets. Nucleic Acids Res. 2019, 47, D607-D613. [CrossRef] [PubMed]

31. Shannon, P.; Markiel, A.; Ozier, O.; Baliga, N.S.; Wang, J.T.; Ramage, D.; Amin, N.; Schwikowski, B.; Ideker, T. Cytoscape: A software environment for integrated models of biomolecular interaction networks. Genome Res. 2003, 13, 2498-2504. [CrossRef]

32. Chin, C.H.; Chen, S.H.; Wu, H.H.; Ho, C.W.; Ko, M.T.; Lin, C.Y. cytoHubba: Identifying hub objects and sub-networks from complex interactome. BMC Syst. Biol. 2014, 8 (Suppl. 4), S11. [CrossRef]

33. Bindea, G.; Galon, J.; Mlecnik, B. CluePedia Cytoscape plugin: Pathway insights using integrated experimental and in silico data. Bioinformatics 2013, 29, 661-663. [CrossRef]

34. Bindea, G.; Mlecnik, B.; Hackl, H.; Charoentong, P.; Tosolini, M.; Kirilovsky, A.; Fridman, W.H.; Pages, F.; Trajanoski, Z.; Galon, J. ClueGO: A Cytoscape plug-in to decipher functionally grouped gene ontology and pathway annotation networks. Bioinformatics 2009, 25, 1091-1093. [CrossRef]

35. Wang, J.; Zhong, J.; Chen, G.; Li, M.; Wu, F.X.; Pan, Y. ClusterViz: A Cytoscape APP for Cluster Analysis of Biological Network. IEEE/ACM Trans. Comput. Biol. Bioinform. 2015, 12, 815-822. [CrossRef]

36. Goeman, J.J. L1 penalized estimation in the Cox proportional hazards model. Biom. J. 2010, 52, 70-84. [PubMed]

37. Hanzelmann, S.; Castelo, R.; Guinney, J. GSVA: Gene set variation analysis for microarray and RNA-seq data. BMC Bioinform. 2013, 14, 7. [CrossRef] [PubMed] 
38. Biswas, B.; Bakhshi, S. Management of Ewing sarcoma family of tumors: Current scenario and unmet need. World J. Orthop. 2016, 7, 527-538. [CrossRef] [PubMed]

39. Simon, N.; Friedman, J.; Hastie, T.; Tibshirani, R. Regularization Paths for Cox's Proportional Hazards Model via Coordinate Descent. J. Stat. Softw. 2011, 39, 1-13. [CrossRef] [PubMed]

40. Gerds, T.A.; Kattan, M.W.; Schumacher, M.; Yu, C. Estimating a time-dependent concordance index for survival prediction models with covariate dependent censoring. Stat. Med. 2013, 32, 2173-2184. [CrossRef]

41. Vickers, A.J.; Cronin, A.M.; Elkin, E.B.; Gonen, M. Extensions to decision curve analysis, a novel method for evaluating diagnostic tests, prediction models and molecular markers. BMC Med. Inform. Decis. Mak. 2008, 8, 53. [CrossRef] [PubMed]

42. Nielsen, T.; Wallden, B.; Schaper, C.; Ferree, S.; Liu, S.; Gao, D.; Barry, G.; Dowidar, N.; Maysuria, M.; Storhoff, J. Analytical validation of the PAM50-based Prosigna Breast Cancer Prognostic Gene Signature Assay and nCounter Analysis System using formalin-fixed paraffin-embedded breast tumor specimens. BMC Cancer 2014, 14, 177. [CrossRef] [PubMed]

43. Stahl, D.; Gentles, A.J.; Thiele, R.; Gutgemann, I. Prognostic profiling of the immune cell microenvironment in Ewing s Sarcoma Family of Tumors. Oncoimmunology 2019, 8, e1674113. [CrossRef] [PubMed]

44. Li, S.; Yang, Q.; Wang, H.; Wang, Z.; Zuo, D.; Cai, Z.; Hua, Y. Prognostic significance of serum lactate dehydrogenase levels in Ewing's sarcoma: A meta-analysis. Mol. Clin. Oncol. 2016, 5, 832-838. [CrossRef] [PubMed] 